\title{
Dorsal Root Ganglion Stimulation for the Treatment of Chronic Pain Syndromes
}

\author{
Natalie H Strand, MD ${ }^{1 *}$, Timothy R. Deer, MD², Ryan S D'Souza MD $^{3}$ \\ ${ }^{1}$ Division of Pain Medicine, Department of Anesthesiology, Mayo Clinic, Arizona. \\ ${ }^{2}$ The Spine and Nerve Center of the Virginia. \\ ${ }^{3}$ Department of Anesthesiology and Perioperative Care, Mayo Clinic, Rochester, Minnesotas. \\ *Corresponding Author: Natalie H. Strand, MD. 5777 E. Mayo Blvd. Phoenix, Arizona 85054, USA.
}

\section{Abstract}

The use of Dorsal Root Ganglion (DRG) stimulation has been an important piece of the algorithm in treating pain related to complex regional pain syndrome (CRPS) types 1 and 2, chronic post-surgical pain, nerve injury, peripheral neuropathies, amputation and other causes of nerve injury. Since its first approval in late 2011 in Europe, this technology has evolved and is currently the best evidence driven therapy for many of the conditions noted in this review. The proper use of the device based on patient selection, spinal anatomy, and physician training is important to capture optimal outcomes that are consistent with the peer-reviewed literature. This publication reviews the key points to offering this therapy to appropriate candidates.

Keywords: Dorsal Root Ganglion Stimulation, DRG Stimulation, Neuromodulation, Complex Regional Pain Syndrome, Spinal Cord Stimulation, Chronic Pain, Neuropathic Pain.

Abbreviations: Dorsal Root Ganglion (DRG), Complex Regional Pain Syndrome (CRPS), Spinal Cord Stimulation (SCS), Wide-dynamic range (WDR)

\section{INTRODUCTION}

The injury of a nerve elicits a complicated neural response at the level of the neuron and at the cellular level in the location of the original injury. This abnormal response sends signals via the peripheral nerve to the dorsal root ganglion (DRG) and then to the spinal cord for processing in the central nervous system. ${ }^{1}$ In normal conditions, this response dissipates with tissue healing and resolution of the original insult. Unfortunately, in conditions such as permanent nerve injury or complex regional pain syndrome (CRPS), the abnormal firing of the peripheral nerve continues despite tissue healing. In these patients, the T-junction, which normally filters the pain signal, becomes hyper-excited and leads to continuing impulses to the dorsal horn neurons in the spinal cord. These sustained DRG discharges have been linked to hyperalgesia. Thus, the DRG is pivotal in the initiation and maintenance of chronic pain. ${ }^{2}$ Animal models have shown a number of changes in these pathways that if left unbridled leads to chronic neuropathic pain. ${ }^{3}$ In addition, rat pain models have shown that the application of electrical current to the DRG greatly reduces the amount of abnormal signaling via the pain neurons traveling to the dorsal horn. ${ }^{4}$ This important mechanistic role of the DRG has made it an attractive anatomical target for pain interruption.

The biochemical mechanism of DRG stimulation is proposed to affect multiple processes implicated in developing and maintaining chronic pain.Stimulation may decrease hyperexcitability of wide-dynamic range (WDR) neurons in the dorsal horn, decrease hyperexcitability of neurons location within the DRG, stabilize peripheral nociceptor sensitization, and stabilize release of neuromodulators and cytokines (e.g. TNF-alpha, chemokines, interleukins, and interferons)..$^{5-7}$ The function of the T-junction is also maintained so that it may filter and reduce propagation of action potentials to the dorsal horn. Effects of DRG stimulation may also transcend at the gene level by normalizing gene expression within the DRG and spinal cord. ${ }^{5}$ 
Dorsal Root Ganglion Stimulation for the Treatment of Chronic Pain Syndromes

\section{Historical Perspective}

The dorsal root ganglion has been a target of clinicians and researchers for many years when examining the treatment of chronic pain. Injection of steroids around the DRG has been used for the treatment of spinal nerve root irritation, post herpetic neuralgia and other sources of nerve injury. ${ }^{8}$ Pulsed and conventional radiofrequency ablation of the DRG has also been used in many areas of the spine including the cervical spine to treat C2 induced nerve pain. Although these treatment options are widespread, they have had a paucity of success in the evidence based evaluation of treatment. The ability to target the DRG using conventional interventional techniques, the clinical response to previous therapies, and the researchbased understanding of this integral structure in pain processing led to an interest in using neuromodulation techniques to stimulate the DRG for the treatment of chronic neuropathic pain conditions.

\section{DEVELOPMENT OF DRG STIMUlation}

In early attempts to stimulate the DRG, the neuromodulation community initially tried to stimulate the fibers by placing a traditional spinal cord stimulation (SCS) lead in the far lateral epidural space or "gutter". Although technically easy, the results were not optimal and outcome data for this method has been unreliable at best. Other attempts at placing traditional SCS leads from a transforaminal approach also failed to produce any success in peer-reviewed evidence based studies. ${ }^{9}$ This lack of success led to innovative approaches and in 2008, Deer and Grigsby became the first clinicians to use novel leads to stimulate the DRG in humans. ${ }^{6}$ Previous data collected from animal and cadaver studies were used to develop a lead to be the correct diameter and shape to correctly stimulate this structure. This novel approach utilized a sheath with a curved tip to be placed into the epidural space which would facilitate placement of the lead over the DRG using a medial to lateral approach, and allow for a special method using loop structures to secure the leads in the epidural space. That initial pilot work led to the feasibility study in which 10 subjects with peripheral nerve related pain syndromes were treated in a short term study with great success. ${ }^{9}$ After this initial success, an international consortium of clinicians and researchers performed a multicenter study showing success in long-term patient followup and a defined response to the on/off use of DRG stimulation in regard to the pain response. ${ }^{10}$ Based on this study and many other diagnosis based successes in the European and Australian experience, the United States, Multicenter, Prospective, Randomized DRG verses Conventional Spinal Cord Stimulation study in CRPS and Causalgia was performed. This study showed superiority of DRG to conventional SCS in both the CRPS and Causalgia groups at both the 3-month and 1-year follow-up period. ${ }^{11}$ This data led to the approval of DRG stimulation via an epidural approach in the United States and led to wider spread use worldwide.

\section{Current Patient Selection}

Based on high-level evidence, patients may benefit from DRG stimulation for conditions in which an abnormal firing of the peripheral nerve is involved in the chronic condition. These conditions include CRPS I, CRPS II, painful peripheral neuropathy, amputationrelated pain syndromes, post-herniorrhaphy nerve pain, chronic postoperative pain (such as total joint replacement or nerve injury), postherpetic neuralgia, and post-mastectomy and post-thoracotomy pain. ${ }^{12}$ The use of DRG stimulation for axial back pain has recently shown great promise in early work. ${ }^{13}$ In addition to selecting the appropriate disease state, the patient should be assessed for proper spinal anatomy and conditions.Severe spinal stenosis, severe foraminal stenosis, or previous surgery at the target level should be seen as relative contraindications. The patient should also be mentally stable and be absent of major untreated depression or anxiety. As with SCS, all medical conditions such as diabetes, bleeding disorders, and active infection should be optimized and addressed prior to the scheduled surgery. In the United States, DRG stimulator leads are approved for placement with the most rostral spinal level of T10, yet it is common for American clinicians to place DRG leads above the T10 level. In Europe and Australia, there are no restrictions to the level of placement. Additionally, experts generally agree that DRG stimulation may be performed up to the $\mathrm{C} 6$ level. ${ }^{14}$

\section{Clinical Outcome Studies Analysis}

\section{Methods}

The authors performed a literature search of the MEDLINE and Google Scholar database through December 27, 2019 using a highly-sensitive text word strategy to find randomized-controlled trials 
Dorsal Root Ganglion Stimulation for the Treatment of Chronic Pain Syndromes

(RCTs), observational studies, and case series/reports discussing use and efficacy of dorsal root ganglion stimulation for any pain condition. Serial searches included terms "dorsalrootganglion stimulation," "DRG stimulation", “DRGstimulator”, “DRGneuromodulation”, "nerve root stimulation", "chronic pain", "complex regional pain syndrome", "chronic postsurgical pain", "peripheral neuropathy", "phantom limb pain", and "failed back surgery syndrome" independently and in combination, using Boolean operators. Authors also performed independent searches of the literature to identify other studies that were not identified in the formal search strategy. Peer-reviewed studies were included for analysis if they provided qualitative or quantitative data on the efficacy of DRG stimulation in the treatment of chronic pain conditions. Studies involving animals, non-English publications, and nonpeer-reviewed articles were excluded.

\section{Complex Regional Pain Syndrome}

There is high-level evidence supporting use of DRG for CRPS. The ACCURATE study was the first RCT which was conducted in 152 patients with CRPS in the lower extremities and compared neurostimulation of the DRG or dorsal column SCS. ${ }^{11}$ The primary endpoint of treatment success was met in this trial with $81.2 \%$ of patients in the DRG arm receiving $\geq 50 \%$ pain relief at three months compared to $55.7 \%$ of patients in the dorsal column SCS arm $(\mathrm{P}<0.001)$. Treatment success persistent at the twelve-month endpoint with $\geq 50 \%$ pain relief reported by $74.2 \%$ in the DRG cohort versus $53.0 \%$ in the SCS cohort $(\mathrm{P}<0.004)$. Other outcomes demonstrated that the DRG cohort reported greater improvement in quality of life and psychological disposition, reported less postural variation in paresthesia, and reported reduced extraneous stimulation in other non-painful areas. There were no differences in device-related or serious adverse outcomes between the two cohorts. Other smaller-scale observational studies by Deer et $\mathrm{al}^{9}$ and Liem et $\mathrm{al}^{10}$ are also consistent with treatment success in CRPS. Future studies are warranted to assess the efficacy of DRG stimulation for CRPS in the upper extremities.

\section{Postsurgical Pain}

In post-herniorrhaphy syndrome, three retrospective observational studies demonstrated neuromodulation of the DRG may be an effective treatment for chronic neuropathic groin pain. ${ }^{15-17}$ Schu et al reported ten of twelve patients with post-herniorrhaphy syndrome had a successful trial of DRG stimulation as defined by at least a $50 \%$ improvement in VAS pain scores. Anecdotally, Schu et al. also reported excellent crossdermatomal paresthesia coverage that even reached discrete pain areas of the groin with use of DRG stimulation. ${ }^{16}$ In post-knee replacement syndrome, one retrospective observational study demonstrated significant reduction in pain scores with use of DRG stimulation. ${ }^{15}$ To date, DRG stimulation for thoracic neuralgia after surgery was shown to be efficacious in case reports of abdominoplasty and bilateral mastectomy. ${ }^{18} \mathrm{~A}$ preliminary case report of DRG stimulation was also reported to treat postthoracotomy syndrome when DRG stimulation was conducted at the precise level of the injury at the T9 level. ${ }^{19}$ Due to the focal nature of pain and the predictability of involved nerves in post-thoracotomy syndrome, this condition may be amenable to DRG stimulation. ${ }^{20}$

\section{Peripheral Neuropathy}

Seven of ten patients in one case series ${ }^{21}$ with lower extremity pain from diabetic peripheral neuropathy experienced significant pain reduction after DRG neuromodulation. There is limited evidence on use of DRG for non-diabetic peripheral neuropathy, such as idiopathic peripheral neuropathy, chemotherapyinduced peripheral neuropathy, and HIV-related neuropathy. ${ }^{14,22}$ DRG stimulation for postherpetic neuralgia has better evidence compared to use of $\mathrm{SCS}^{23,24}$

\section{Phantom Limb Pain}

Limited evidence is present on use of DRG for phantom limb pain and may be considered in select patients. The largest case series consisted of eight patients, of which four of six patients with lower extremity amputation and one of two patients with upper extremity amputation reported significant pain relief at a mean follow-up period of 14.4 months. $^{25}$

\section{Failed Back Surgery Syndrome}

Contrary to the aforementioned pain syndromes, SCS remains advantageous compared to DRG neuromodulation in treating failed back surgery syndrome (FBSS). In a prospective observational study of sixteen patients with FBSS, only eight patients had successful DRG trials and received a permanent implant. Furthermore, a successful 
Dorsal Root Ganglion Stimulation for the Treatment of Chronic Pain Syndromes

response rate specifically in the back as defined by at least a $50 \%$ reduction in back pain VAS score was only observed in $37.5 \%$ of patients. ${ }^{26}$ It is possible that epidural scar tissue in FBSS may make placement of DRG leads challenging and may increase stimulation impedance. $^{27}$

\section{Physician Training AND Procedure OVERVIEW}

In experienced hands, DRG stimulation trials and permanent implants have been shown to be as safe as SCS and more efficacious in many patient groups. ${ }^{28}$ Physicians utilizing this therapy should be taught to understand the anatomical goals of DRG stimulation during pain medicine fellowship or in DRG-specific training sessions. This involves assessing the preoperative imaging to get a plan of implant, and executing the steps of the procedure. Prior to the trial, preoperative images should be reviewed to assess any possible hindrance to lead placement and to allow the physician to develop a plan for the needle angle that can best deliver the sheath to the appropriate anatomical location. The patient is prone with pillows placed under the abdomen to minimize lumbar lordosis. After the patient is properly prepped and draped, a 14-gauge needle is used to access the epidural space from an inferior and contralateral approach. Once in the epidural space, the sheath and stimulation lead are advanced to the area just below the pedicle of the desired level. The lead is advanced through the foramen over the DRG. Often, a "pop" is felt as the lead advances through the foraminal ligaments. After the lead is in proper position under an antero-posterior view, a lateral view should be obtained to confirm dorsal placement. Next, the sheath is manipulated to allow for an S loop to be left in the epidural space as a means of reducing lead migration. For a trial, leads are then secured to the skin similar to dorsal column SCS trials. For permanent implantation, the lead should be secured to the fascia and then tunneled subcutaneously to the implantable permanent generator.

\section{DRG SAFETY}

A recent analysis of FDA required reporting data from the manufacturer of DRG showed the safety to be equal to that of conventional SCS. ${ }^{29}$ This was true for all categories of adverse events. The most common adverse events reported from DRG systems included infection $(1.08 \%)$, cerebrospinal fluid leak $(0.54 \%)$, and persistent pain at the implant site $(0.18 \%) .{ }^{29} \mathrm{This}$ is consistent with the data from the ACCURRATE study that led to the IDE approval analysis.

\section{FUTURE VIEWS AND OPTIONS ON DRG STIMULATION}

Thefuture of DRG stimulation willbe impactful. Current development involves a DRG paddle lead that will allow patients with difficult spinal anatomy and previous surgery to access this important treatment. In addition, novel waveforms are being added to current software options. The use of these new waveforms may be helpful to maintain therapy and avoid the pitfall of therapy tolerance. Another potential major advancement is the use of neuro-monitoring under general anesthesia to place DRG leads. This leads to an immobile patient and real time monitoring of safety during lead placement. ${ }^{30}$

\section{ConClusion}

Since the approval of DRG stimulation in the United States, The European Union, and Australia, thousands of patients have benefitted. Continued vigilance in patient selection, technique, and long-term solutions for patients who suffer from severe pain syndromes will lead to the desired long term results with this therapy.

\section{REFERENCES}

[1] Pope JE, Deer TR, Kramer J. A systematic review: current and future directions of dorsal root ganglion therapeutics to treat chronic pain. Pain Med. 2013;14(10):1477-1496.

[2] Liem L, van Dongen E, Huygen FJ, Staats P, Kramer J. The Dorsal Root Ganglion as a Therapeutic Target for Chronic Pain. Reg Anesth Pain Med. 2016;41(4):511-519.

[3] Malik K, Benzon HT. Radiofrequency applications to dorsal root ganglia: a literature review. Anesthesiology. 2008;109(3):527-542.

[4] Perret DM, Kim DS, Li KW, et al. Application of pulsed radiofrequency currents to rat dorsal root ganglia modulates nerve injury-induced tactile allodynia. Anesth Analg. 2011;113(3):610-616.

[5] Deer TR, Jain S, Hunter C, Chakravarthy K. Neurostimulation for Intractable Chronic Pain. Brain Sci. 2019;9(2).

[6] Croom JE, Foreman RD, Chandler MJ, Barron KW. Cutaneous vasodilation during dorsal column stimulation is mediated by dorsal roots and CGRP. Am J Physiol. 1997;272(2 Pt 2):H950-957. 
Dorsal Root Ganglion Stimulation for the Treatment of Chronic Pain Syndromes

[7] Koopmeiners AS, Mueller S, Kramer J, Hogan QH. Effect of electrical field stimulation on dorsal root ganglion neuronal function. Neuromodulation. 2013;16(4):304-311; discussion 310-301.

[8] Fujiwara A, Watanabe K, Hashizume K, Shinohara K, Kawaguchi M. Transforaminal vs Interlaminar Epidural Steroid Injection for Acute-Phase Shingles: A Randomized, Prospective Trial. Pain Physician. 2018;21(4):373-382.

[9] Deer TR, Grigsby E, Weiner RL, Wilcosky B, Kramer JM. A prospective study of dorsal root ganglion stimulation for the relief of chronic pain. Neuromodulation. 2013;16(1):67-71; discussion 71-62.

[10] Liem L, Russo M, Huygen FJ, et al. A multicenter, prospective trial to assess the safety and performance of the spinal modulation dorsal root ganglion neurostimulator system in the treatment of chronic pain. Neuromodulation. 2013;16(5):471-482; discussion 482.

[11] Deer TR, Levy RM, Kramer J, et al. Dorsal root ganglion stimulation yielded higher treatment success rate for complex regional pain syndrome and causalgia at 3 and 12 months: a randomized comparative trial. Pain. 2017;158(4):669-681.

[12] Esposito MF, Malayil R, Hanes M, Deer T. Unique Characteristics of the Dorsal Root Ganglion as a Target for Neuromodulation. Pain Med. 2019;20(Suppl 1):S23-S30.

[13] Chapman KB, Groenen PS, Patel KV, Vissers KC, van Helmond N. T12 Dorsal Root Ganglion Stimulation to Treat Chronic Low Back Pain: A Case Series. Neuromodulation. 2019.

[14] Deer TR, Pope JE, Lamer TJ, et al. The Neuromodulation Appropriateness Consensus Committee on Best Practices for Dorsal Root Ganglion Stimulation. Neuromodulation. 2019;22(1):1-35.

[15] Hunter CW, Sayed D, Lubenow T, et al. DRG FOCUS: A Multicenter Study Evaluating Dorsal Root Ganglion Stimulation and Predictors for Trial Success. Neuromodulation. 2019;22(1):61-79.

[16] Schu S, Gulve A, ElDabe S, et al. Spinal cord stimulation of the dorsal root ganglion for groin pain-a retrospective review. Pain Pract. 2015;15(4):293-299.
[17] Zuidema X, Breel J, Wille F. Paresthesia mapping: a practical workup for successful implantation of the dorsal root ganglion stimulator in refractory groin pain. Neuromodulation. 2014;17(7):665669; discussion 669.

[18] Anthony CL, Tora MS, Bentley JN, Texakalidis P, Boulis NM. Dorsal Root Ganglion Stimulation for Thoracic Neuralgia: A Report of Six Cases. Cureus. 2019;11(5):e4615.

[19] Ali R, Epstein L, Khelemsky Y. Successful treatment of chronic post thoracotomy pain syndrome with dorsal root ganglion (DRG) stimulation. Paper presented at: 20th Annual North American Neuromodulation SocietyJuly 19-22, 2017; Las Vegas, NV.

[20] Antony AB, Schultheis BC, Jolly SM, Bates D, Hunter CW, Levy RM. Neuromodulation of the Dorsal Root Ganglion for Chronic Postsurgical Pain. Pain Med. 2019;20(Suppl 1):S41-S46.

[21] Eldabe S, Espinet A, Wahlstedt A, et al. Retrospective Case Series on the Treatment of Painful Diabetic Peripheral Neuropathy With Dorsal Root Ganglion Stimulation. Neuromodulation. 2018;21(8):787-792.

[22] Falowski S, Pope JE, Raza A. Early US Experience With Stimulation of the Dorsal Root Ganglia for the Treatment of Peripheral Neuropathy in the Lower Extremities: A Multicenter Retrospective Case Series. Neuromodulation. 2019;22(1):96-100.

[23] Piedade GS, Vesper J, Chatzikalfas A, Slotty PJ. Cervical and High-Thoracic Dorsal Root Ganglion Stimulation in Chronic Neuropathic Pain. Neuromodulation. 2019;22(8):951-955.

[24] Yanamoto F, Murakawa K. The effects of temporary spinal cord stimulation (or spinal nerve root stimulation) on the management of early postherpetic neuralgia from one to six months of its onset. Neuromodulation. 2012;15(2):151154; discussion 154.

[25] Eldabe S, Burger K, Moser H, et al. Dorsal Root Ganglion (DRG) Stimulation in the Treatment of Phantom Limb Pain (PLP). Neuromodulation. 2015;18(7):610-616; discussion 616-617.

[26] Liem L, Russo M, Huygen FJ, et al. One-year outcomes of spinal cord stimulation of the dorsal root ganglion in the treatment of chronic neuropathic pain. Neuromodulation. 2015;18(1):41-48; discussion 48-49. 
Dorsal Root Ganglion Stimulation for the Treatment of Chronic Pain Syndromes

[27] Grill WM, Mortimer JT. Electrical properties of implant encapsulation tissue. Ann Biomed Eng. 1994;22(1):23-33.

[28] Deer T. Dorsal root ganglion safety analysis of the U.S. experience. Paper presented at: WVSIPP meeting presentation 2018.

[29] Deer T, Pope J, Hunter C, et al. Safety Analysis of Dorsal Root Ganglion Stimulation in the Treatment of Chronic Pain. Neuromodulation. 2019.
[30] Falowski SM, Dianna A. A Prospective Analysis of Neuromonitoring for Confirmation of Lead Placement in Dorsal Root Ganglion Stimulation. Oper Neurosurg (Hagerstown). 2018;14(6):654-660.

Citation: Natalie H Strand, Timothy R. Deer, Ryan S D'Souza. Dorsal Root Ganglion Stimulation for the Treatment of Chronic Pain Syndromes. Archives of Anesthesiology. 2020; 3(2): 01-06

Copyright: (C) 2020 : Natalie H Strand, Timothy R. Deer, Ryan S D'Souza This is an open access article distributed under the Creative Commons Attribution License, which permits unrestricted use, distribution, and reproduction in any medium, provided the original work is properly cited. 